\title{
Role of syntrophic microbial communities in high-rate methanogenic bioreactors
}

\author{
Alfons J. M. Stams, Diana Z. Sousa, Robbert Kleerebezem \\ and Caroline M. Plugge
}

\begin{abstract}
$\overline{\text { ABSTRACT }}$
Anaerobic purification is a cost-effective way to treat high strength industrial wastewater. Through anaerobic treatment of wastewaters energy is conserved as methane, and less sludge is produced. For high-rate methanogenesis compact syntrophic communities of fatty acid-degrading bacteria and methanogenic archaea are essential. Here, we describe the microbiology of syntrophic communities in methanogenic reactor sludges and provide information on which microbiological factors are essential to obtain high volumetric methane production rates. Fatty-acid degrading bacteria have been isolated from bioreactor sludges, but also from other sources such as freshwater sediments. Despite the important role that fatty acid-degrading bacteria play in high-rate methanogenic bioreactors, their relative numbers are generally low. This finding indicates that the microbial community composition can be further optimized to achieve even higher rates.
\end{abstract}

Key words | anaerobic treatment, granulation, methanogenesis, syntrophic communities, UASB reactor



\section{ANAEROBIC WASTEWATER TREATMENT}

Anaerobic treatment is one of the most cost-effective ways to treat industrial wastewater with high organic matter content (Kosaric \& Blaszczyk I990; Lettinga I995; van Lier et al. 200I). In particular, the introduction of the Upflow Anaerobic Sludge Blanket (UASB) reactor, over three decades ago, has resulted in improved treatment of industrial wastewaters. In a UASB reactor the wastewater is pumped from the bottom into the reactor, and is purified while passing a bed of compact biomass (granular sludge), which is formed by self-immobilization of anaerobic bacteria and methanogenic archaea (Lettinga et al. I980; van Lier et al. 200I). The high density of these granules prevents microorganisms from being washed out, while the short intermicrobial distances are favourable for the transfer of metabolites from the bacteria to the methanogens. A variety of additional anaerobic bioreactor designs has been developed. Expanded Granular Sludge Blanket (EGSB) and Internal Circulation (IC) reactors have replaced the more conventional UASB systems (Franklin 200I). EGSB systems have a comparable design to UASB reactors, but contain an expanded granular sludge bed minimizing external mass doi: $10.2166 /$ wst.2012.192 transfer limitations around the biofilm. Anaerobic treatment systems can operate at different temperatures and are suited for different types of wastewater. Currently, anaerobic treatment technologies are widely applied to treat wastewaters from food processing industries, pulp and paper industries and chemical and petrochemical industries (Lettinga I995; Macarie 2000).

\section{METHANOGENESIS}

Physiologically and phylogenetically different microbial groups are involved in the mineralization of complex organic matter to methane and carbon dioxide (Gujer \& Zehnder I983; de Bok et al. 2004; Stams et al. 2006). In general, biopolymers including proteins, carbohydrates, nucleic acids and fats are first hydrolyzed to mono- and oligomers, and these are then fermented to products that can be used by methanogens directly (acetate, hydrogen, formate) and to other fatty acids such as propionate, butyrate, branchedchain fatty acids and long-chain fatty acids (Gujer \& 
Zehnder 1983). Acetogenic bacteria oxidize these fatty acids anaerobically to acetate, $\mathrm{CO}_{2}, \mathrm{H}_{2}$ and formate (Gujer \& Zehnder I983; Stams \& Plugge 2009). Generally, about $70 \%$ of the methane is formed from acetate, while the remainder is formed from $\mathrm{H}_{2} / \mathrm{CO}_{2}$ and formate (Gujer \& Zehnder I983; Conrad 1999). However, acetate can also be degraded by syntrophic communities of bacteria and archaea, resulting in a more prominent role of $\mathrm{H}_{2} / \mathrm{CO}_{2}$ and formate as methanogenic substrates (Hattori 2008). In mesophilic digesters, high ammonia levels can cause syntrophic acetate oxidation (Westerholm et al. 20Io). The ammonia tolerance of these syntrophic acetate oxidizers gives them a competitive advantage in ammonia-stressed systems. These bacteria, in association with ammonia-tolerant hydrogenotrophic methanogens, may consequently adopt the role of dominant acetate consumers in environments in which ammonia restrains aceticlastic methanogenic activity (Westerholm et al. 20II).

Propionate and butyrate are key intermediates in the mineralization of complex organic matter. The complete oxidation of propionate and butyrate can account for 20$43 \%$ of the total methane formation, depending on the type of digester and the nature of the organic compounds (Mackie \& Bryant I98I).

\section{SYNTROPHIC FATTY ACID DEGRADATION}

Easily degradable compounds (soluble polysaccharides, proteins, sugars and amino acids) in industrial wastewater are rapidly degraded by primary fermenters during storage and the passage to the anaerobic bioreactor. Therefore, the main substrates that enter the anaerobic bioreactor are mixtures of fatty acids, mainly acetate, propionate and butyrate. Propionate and butyrate are important intermediates in methanogenesis. They are formed in the anaerobic fermentation of polysaccharides and proteins (Stams I994; Schink \& Stams 2006). Typically these fatty acids are further degraded by syntrophic associations of anaerobic bacteria and methanogenic archaea, which are dependent on each other for energy conservation and growth (McInerney et al. 2008; Sousa et al. 2009; Stams \& Plugge 2009). For complete and high-rate methanogenesis such syntrophic communities are indispensable.

Bacteria that degrade and grow on fatty acids have to cope with the unfavourable energetics of the conversion processes. Table 1 illustrates the conversion of propionate and butyrate to the methanogenic substrates acetate, hydrogen and formate. It is evident that bacteria can only derive
Table 1 | Standard Gibbs free energy changes of reactions involved in syntrophic propionate and butyrate degradation. Data from Thauer et al. (1977)

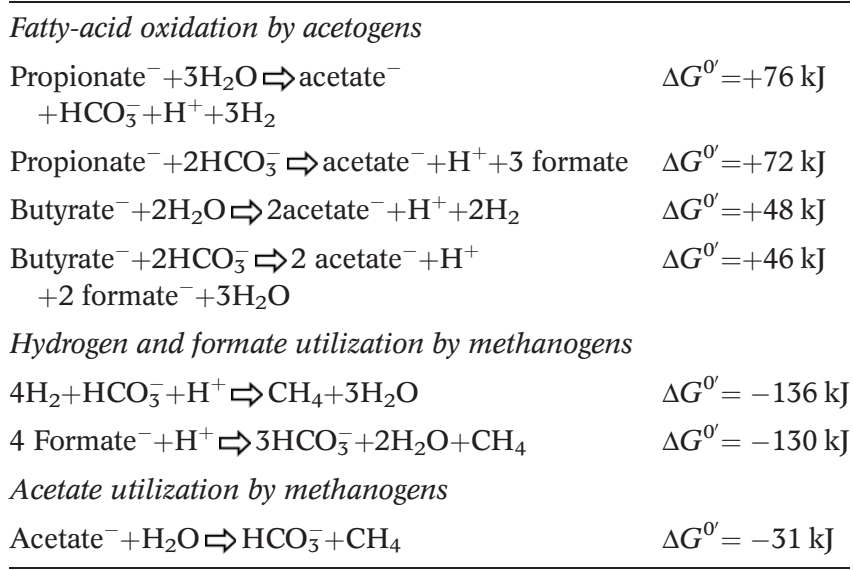

energy for growth from these conversions when the concentration of the products (especially hydrogen and formate) is kept low. This results in an obligate dependence of acetogenic bacteria on methanogenic archaea. Obligately syntrophic communities of propionate- and butyrate-degrading acetogenic bacteria and methanogenic archaea have several unique features: they degrade fatty acids coupled to growth, while neither the bacterium nor the methanogen alone is able to degrade these compounds; intermicrobial distances influence biodegradation rates and specific growth rates, which in methanogenic bioreactors results in the formation of aggregates of bacteria and archaea (granular sludge); the syntrophic communities grow in conditions that are close to thermodynamical equilibrium, and the communities have evolved biochemical mechanisms that allow sharing of chemical energy (Stams \& Plugge 2009). There is still discussion on whether hydrogen and formate are the primary compounds for interspecies electron transfer, and it is still unclear what their relative importance is (Stams \& Plugge 2009; Müller et al. 20Io; Worm et al. 20II).

The reported maximum specific growth rates of propionate- and butyrate-degrading acetogenic bacteria in suspended co-culture with methanogens were 0.10 and 0.19 day $^{-1}$, respectively (McInerney et al. I979; Boone \& Bryant I980; Mountfort \& Bryant I982). It is evident that fatty acid-degrading bacteria grow slowly, but it is not clear fully how fast they can grow in tight physical aggregation with methanogens. In fact, specific growth rates of syntrophic communities are difficult to determine as the conversion rate per cell is not constant, but is dependent on cell density of the partner microorganism. 


\section{Syntrophic propionate-degrading bacteria}

Boone \& Bryant (1980) described Syntrophobacter wolinii, a propionate-degrading bacterium that grows in syntrophic association with methanogens or sulfate-reducing bacteria (Table 2). Several other mesophilic and thermophilic bacteria that grow in syntrophy with methanogens have been described since then as reviewed by McInerney et al. (2008). These include Gram-negative bacteria (Syntrophobacter and Smithella) and Gram-positive bacteria (Pelotomaculum and Desulfotomaculum). Both groups are phylogenetically related to sulfate-reducing bacteria and some species are also able to use sulfate as a terminal electron acceptor for growth. Syntrophobacter and Desulfotomaculum species reduce sulfate, but Smithella and Pelotomaculum cannot reduce sulfate. Most syntrophic propionate-degrading bacteria are able to grow in pure culture by fermentation of fumarate or pyruvate. Fermentative growth or sulfate-dependent growth was used to obtain the bacteria in pure culture. The only exceptions are Pelotomaculum schinkii (de Bok et al. 2005) and P. propionicicum (Imachi et al. 2007), which seem to be true propionatedegrading syntrophs. $P$. thermopropionicum and Desulfotomaculum thermobenzoicum (subsp. thermopropionicum) are moderately thermophilic and grow in syntrophy with thermophilic methanogens (Imachi et al. 2002; Plugge et al. 2002). A marine propionate-degrading syntrophic community has been described, but the identity of the propionate-degrading bacterium is not known (Kendall et al. 2006). In sulfate-rich environments sulfate reduction is the main terminal process and several sulfate reducers that degrade propionate have been described. Therefore, in marine habitats syntrophic growth on propionate seems to be restricted to sulfate-depleted zones.

Two pathways for propionate metabolism are known, the methylmalonyl-CoA pathway and a dismutation pathway. In the latter pathway two propionate molecules are converted to acetate and butyrate, the butyrate being degraded to acetate and hydrogen as described below. Thus far, this pathway is only found in Smithella propionica (Liu et al. I999; de Bok et al. 200I). The methylmalonyl-CoA pathway is found in the other syntrophic propionate-oxidizing bacteria (McInerney et al. 2008). In the methylmalonyl-CoA pathway, propionate is first activated to propionyl-CoA and then carboxylated to methylmalonyl-CoA. Methylmalonyl-CoA is rearranged to form succinyl-CoA, which is converted to succinate. Succinate is oxidized to fumarate, which is then hydrated to malate and oxidized to oxaloacetate. Pyruvate is formed by decarboxylation, and is further oxidized in a
HS-CoA-dependent decarboxylation to acetyl-CoA and finally to acetate.

\section{Syntrophic butyrate- and LCFA-degrading bacteria}

McInerney et al. (198I) enriched and characterized Syntrophomonas wolfei, a Gram-positive bacterium that degrades butyrate and some other short-chain fatty acids in syntrophic association with methanogens (Table 2). Several other bacteria have been described that grow with butyrate or higher fatty acids in syntrophy with methanogens or sulfate reducers as hydrogen scavengers (McInerney et al. 2008; Sousa et al. 2009). Thus far, Algorimarina butyrica is the only psychrophilic and marine bacterium that is known to degrade butyrate in syntrophy with methanogens (Kendall et al. 2006). This bacterium is not able to grow with other short-chain fatty acids like valerate or caproate or longer chain fatty acids such as palmitate. Mesophilic bacteria capable of syntrophic fatty acid metabolism are mainly species of Syntrophomonas, though $S$. bryantii was previously named Syntrophospora bryantii and Clostridium bryantii (Stieb \& Schink 1985; Zhao et al. 1990; Wu et al. 2006a; Sousa et al. 2009). The only exception is

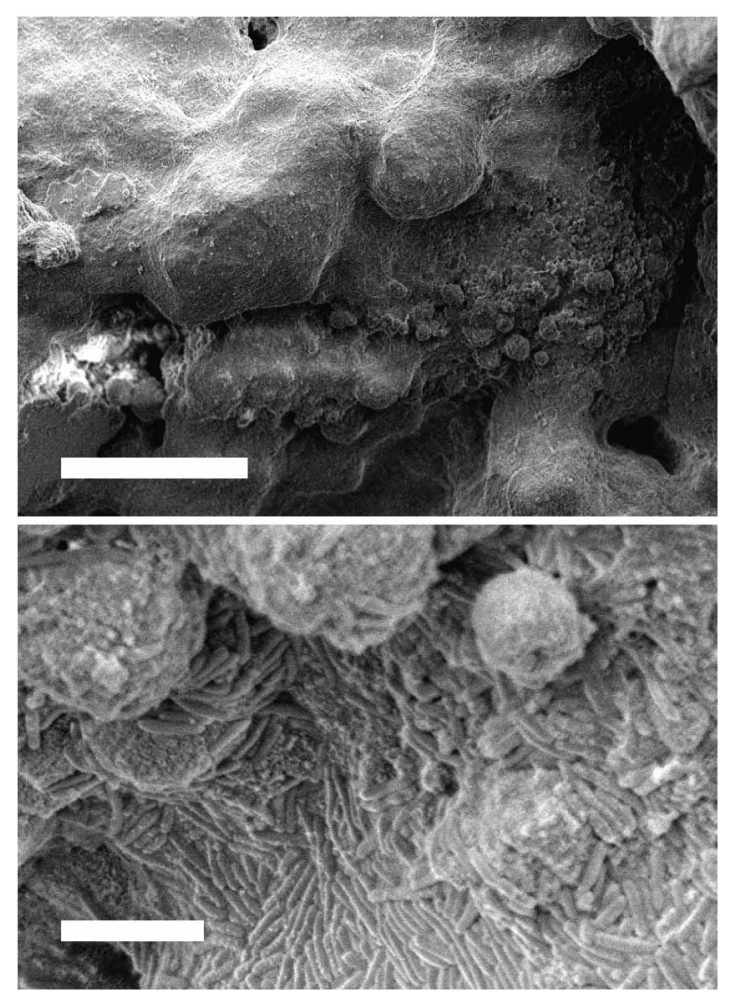

Figure 1 | Scanning electron microscopic (SEM) images that show embedded microcolonies in an anaerobic methanogenic granule. The bar represents $50 \mu \mathrm{M}$ (upper panel) and $5 \mu \mathrm{M}$ (lower panel). 
Table 2 | Isolation source of fatty-acid degrading bacteria (in grey: bacteria isolated from anaerobic reactor sludge) and the respective methanogenic partner

\begin{tabular}{|c|c|c|c|c|}
\hline Syntrophic bacterium & Syntrophic partner & $\begin{array}{l}\text { Substrates used } \\
\text { in co-culture }\end{array}$ & Isolated from & References \\
\hline $\begin{array}{l}\text { Syntrophobacter } \\
\text { fumaroxidans }\end{array}$ & $\begin{array}{l}\text { Methanospirillum } \\
\text { hungatei }\end{array}$ & $\mathrm{C}_{3}$ & $\begin{array}{l}\text { Granular sludge from a full-scale } \\
\text { UASB reactor treating sugar beet } \\
\text { waste, Breda, The Netherlands }\end{array}$ & Harmsen et al. (1998) \\
\hline $\begin{array}{l}\text { Syntrophobacter } \\
\text { pfennigii }\end{array}$ & $\begin{array}{l}\text { Methanospirillum } \\
\text { hungatei }\end{array}$ & $\mathrm{C}_{3}$ & $\begin{array}{l}\text { Anoxic sludge of a municipal } \\
\text { sewage plant in Konstanz, } \\
\text { Germany }\end{array}$ & $\begin{array}{l}\text { Wallrabenstein et al. } \\
\text { (I995) }\end{array}$ \\
\hline $\begin{array}{l}\text { Syntrophobacter } \\
\text { sulfatireducens }\end{array}$ & $\begin{array}{l}\text { Methanospirillum } \\
\text { hungatei }\end{array}$ & $\mathrm{C}_{3}$ & $\begin{array}{l}\text { Granular sludge from a UASB } \\
\text { reactor treating brewery } \\
\text { wastewater in Beijing, China }\end{array}$ & Chen et al. (2005) \\
\hline $\begin{array}{l}\text { Syntrophobacter } \\
\text { wolinii }\end{array}$ & $\begin{array}{l}\text { Methanospirillum } \\
\text { hungatei } \\
\text { Desulfovibrio sp. }\end{array}$ & $\mathrm{C}_{3}$ & $\begin{array}{l}\text { Anaerobic sewage digester sludge in } \\
\text { Illinois, USA }\end{array}$ & Boone \& Bryant (1980) \\
\hline $\begin{array}{l}\text { Pelotomaculum } \\
\text { schinkii }\end{array}$ & $\begin{array}{l}\text { Methanospirillum } \\
\text { hungatei }\end{array}$ & $\mathrm{C}_{3}$ & $\begin{array}{l}\text { Granular sludge from a full-scale } \\
\text { UASB reactor treating sugar beet } \\
\text { waste, Breda, The Netherlands }\end{array}$ & de Bok et al. (2005) \\
\hline $\begin{array}{l}\text { Pelotomaculum } \\
\text { thermopropionicum }\end{array}$ & $\begin{array}{c}\text { Methanothermobacter } \\
\text { themautotrophicus }\end{array}$ & $\mathrm{C}_{3}$ & $\begin{array}{l}\text { Granular sludge from a } \\
\text { thermophilic laboratory-scale } \\
\text { UASB reactor, Japan }\end{array}$ & Imachi et al. (2002) \\
\hline $\begin{array}{l}\text { Pelotomaculum } \\
\text { propionicicum }\end{array}$ & $\begin{array}{l}\text { Methanospirillum } \\
\text { hungatei }\end{array}$ & $\mathrm{C}_{3}$ & $\begin{array}{l}\text { Granular sludge from a mesophilic } \\
\text { laboratory-scale UASB reactor, } \\
\text { Japan }\end{array}$ & Imachi et al. (2007) \\
\hline Smithella propionica & $\begin{array}{l}\text { Methanospirillum } \\
\text { hungatei } \\
\text { Methanogenium sp. }\end{array}$ & $\mathrm{C}_{3}, \mathrm{C}_{4}$ & $\begin{array}{l}\text { Granular sludge from a } \\
\text { mesophilic laboratory-scale } \\
\text { UASB reactor, USA }\end{array}$ & Liu et al. (I999) \\
\hline $\begin{array}{l}\text { Desulfotomaculum } \\
\text { thermobenzoicum } \\
\text { thermosyntrophicum }\end{array}$ & $\begin{array}{r}\text { Methanothermobacter } \\
\text { thermautotrophicus }\end{array}$ & $\mathrm{C}_{3}, \mathrm{C}_{4}$ & $\begin{array}{l}\text { Granular sludge from a } \\
\text { thermophilic laboratory-scale } \\
\text { UASB reactor, } \\
\text { The Netherlands }\end{array}$ & Plugge et al. (2002) \\
\hline $\begin{array}{l}\text { Desulfotomaculum } \\
\text { thermocisternum }\end{array}$ & $\begin{array}{l}\text { Methanococcus } \\
\text { thermolithotrophicus }\end{array}$ & $\mathrm{C}_{3}, \mathrm{C}_{4}$ & $\begin{array}{l}\text { North Sea oil reservoir formation } \\
\text { water, Norway }\end{array}$ & Nilsen et al. (1996) \\
\hline Algorimarina butyrica & Methanogenium sp. & $\mathrm{C}_{4}$ & $\begin{array}{l}\text { Permanently cold, shallow anoxic } \\
\text { marine sediments in Skan Bay, } \\
\text { Alaska }\end{array}$ & Kendall et al. (2006) \\
\hline $\begin{array}{l}\text { Syntrophomonas } \\
\text { bryantii }\end{array}$ & $\begin{array}{l}\text { Methanospirillum } \\
\text { hungatei } \\
\text { Desulfovibrio sp. }\end{array}$ & $\mathrm{C}_{4}-\mathrm{C}_{11}$ & $\begin{array}{l}\text { Marine anoxic mud taken } \\
\text { near Cuxhaven, mud of } \\
\text { a creek near Konstanz, } \\
\text { anaerobic digester sludge, } \\
\text { Germany }\end{array}$ & $\begin{array}{l}\text { Stieb \& Schink (1985); } \\
\text { Zhao et al. (1990); } \\
\text { Wu et al. (2006a) }\end{array}$ \\
\hline $\begin{array}{l}\text { Syntrophomonas } \\
\text { cellicola }\end{array}$ & $\begin{array}{l}\text { Methanobacterium } \\
\text { formicicum } \\
\text { Desulfovibrio sp. }\end{array}$ & $\mathrm{C}_{4}-\mathrm{C}_{8}, \mathrm{C}_{10}$ & $\begin{array}{l}\text { Sample from the walls of a distilled- } \\
\text { spirit fermenting cellar in Hebei } \\
\text { province, China }\end{array}$ & Wu et al. (2006a) \\
\hline $\begin{array}{l}\text { Syntrophomonas } \\
\text { curvata }\end{array}$ & $\begin{array}{l}\text { Methanobacterium } \\
\text { formicicum }\end{array}$ & $\mathrm{C}_{4}-\mathrm{C}_{18}, \mathrm{C}_{18: 1}$ & $\begin{array}{l}\text { Granular sludge from a UASB } \\
\text { reactor treating brewery } \\
\text { wastewater in Beijing, China }\end{array}$ & $\begin{array}{l}\text { Zhang et al. (2004; } \\
\text { 2005) }\end{array}$ \\
\hline $\begin{array}{l}\text { Syntrophomonas erecta } \\
\text { erecta }\end{array}$ & $\begin{array}{l}\text { Methanospirillum } \\
\text { hungatei }\end{array}$ & $\mathrm{C}_{4}-\mathrm{C}_{8}$ & $\begin{array}{l}\text { Granular sludge of a UASB reactor } \\
\text { treating bean-curd farm } \\
\text { wastewater in Beijing, China }\end{array}$ & $\begin{array}{l}\text { Zhang et al. (2005); } \\
\text { Wu et al. (2006b) }\end{array}$ \\
\hline $\begin{array}{l}\text { Syntrophomonas erecta } \\
\text { sporosyntropha }\end{array}$ & $\begin{array}{l}\text { Methanobacterium } \\
\text { formicicum }\end{array}$ & $\mathrm{C}_{4}-\mathrm{C}_{8}$ & $\begin{array}{l}\text { River sediment in Beijing and rice } \\
\text { field mud in Yunnan Province, } \\
\text { China }\end{array}$ & Wu et al. (2006b) \\
\hline
\end{tabular}


Table 2 | continued

\begin{tabular}{|c|c|c|c|c|}
\hline Syntrophic bacterium & Syntrophic partner & $\begin{array}{l}\text { Substrates used } \\
\text { in co-culture }\end{array}$ & Isolated from & References \\
\hline $\begin{array}{l}\text { Syntrophomonas } \\
\text { palmitatica }\end{array}$ & $\begin{array}{l}\text { Methanospirillum } \\
\text { hungatei }\end{array}$ & $\mathrm{C}_{4}-\mathrm{C}_{18}$ & $\begin{array}{l}\text { Granular sludge from a laboratory- } \\
\text { scale UASB reactor treating palm } \\
\text { oil mill effluent, Japan }\end{array}$ & Hatamoto et al. (2007) \\
\hline $\begin{array}{l}\text { Syntrophomonas } \\
\text { saponavida }\end{array}$ & $\begin{array}{l}\text { Methanospirillum } \\
\text { hungatei } \\
\text { Desulfovibrio sp. }\end{array}$ & $\mathrm{C}_{4}-\mathrm{C}_{18}$ & $\begin{array}{l}\text { Anaerobic sewage digester sludge in } \\
\text { Illinois, USA }\end{array}$ & $\begin{array}{l}\text { Lorowitz et al. (1989); } \\
\text { Wu et al. (2007) }\end{array}$ \\
\hline $\begin{array}{l}\text { Syntrophomonas } \\
\text { sapovorans }\end{array}$ & $\begin{array}{l}\text { Methanospirillum } \\
\text { hungatei }\end{array}$ & $\begin{array}{l}\mathrm{C}_{4}-\mathrm{C}_{18}, \mathrm{C}_{16: 1}, \mathrm{C}_{18: 1} \\
\quad \mathrm{C}_{18: 2}\end{array}$ & $\begin{array}{l}\text { Sewage digester sludge in } \\
\text { Marquette-Lez-Lille, France }\end{array}$ & $\begin{array}{l}\text { Roy et al. (I986); } \\
\text { Zhang et al. (2005) }\end{array}$ \\
\hline $\begin{array}{l}\text { Syntrophomonas } \\
\quad \text { wolfei } \\
\text { methylbutyratica }\end{array}$ & $\begin{array}{l}\text { Methanobacterium } \\
\text { formicicum }\end{array}$ & $\mathrm{C}_{4}-\mathrm{C}_{8}$ & $\begin{array}{l}\text { Rice field mud in Jangxi province, } \\
\text { China }\end{array}$ & Wu et al. (2007) \\
\hline $\begin{array}{l}\text { Syntrophomonas } \\
\text { wolfei wolfei }\end{array}$ & $\begin{array}{l}\text { Methanospirillum } \\
\text { hungatei } \\
\text { Desulfovibrio sp. }\end{array}$ & $\mathrm{C}_{4}-\mathrm{C}_{8}$ & $\begin{array}{l}\text { Sewage digester sludge and rumen } \\
\text { digester, USA }\end{array}$ & $\begin{array}{l}\text { McInerney et al. (I979; } \\
\text { I98I); } \\
\text { Zhang et al. (2005) }\end{array}$ \\
\hline $\begin{array}{l}\text { Syntrophomonas } \\
\text { zehnderi }\end{array}$ & $\begin{array}{l}\text { Methanobacterium } \\
\text { formicicum }\end{array}$ & $\begin{array}{l}\mathrm{C}_{4}-\mathrm{C}_{18}, \mathrm{C}_{16: 1}, \mathrm{C}_{18: 1}, \\
\quad \mathrm{C}_{18: 2}\end{array}$ & $\begin{array}{l}\text { Anaerobic sludge from an EGSB } \\
\text { treating oleate-based effluent, } \\
\text { Braga, Portugal }\end{array}$ & Sousa et al. (2007) \\
\hline $\begin{array}{l}\text { Syntrophus } \\
\text { aciditrophicus }\end{array}$ & $\begin{array}{l}\text { Methanospirillum } \\
\text { hungatei } \\
\text { Desulfovibrio sp. }\end{array}$ & $\mathrm{C}_{4}-\mathrm{C}_{8}, \mathrm{C}_{16}, \mathrm{C}_{18}$ & $\begin{array}{l}\text { Sewage sludge from a municipal } \\
\text { sewage treatment plant in } \\
\text { Norman, Oklahoma, USA }\end{array}$ & Jackson et al. (1999) \\
\hline $\begin{array}{l}\text { Syntrophothermus } \\
\text { lipocalidus }\end{array}$ & $\begin{array}{l}\text { Methanothermobacter } \\
\text { thermoautotrophicus }\end{array}$ & $\mathrm{C}_{4}-\mathrm{C}_{10}$ & $\begin{array}{l}\text { Granular sludge from a laboratory- } \\
\text { scale thermophilic UASB reactor, } \\
\text { Japan }\end{array}$ & Sekiguchi et al. (2000) \\
\hline $\begin{array}{l}\text { Thermosyntropha } \\
\quad \text { lipolytica }\end{array}$ & Methanobacterium sp. & $\mathrm{C}_{4}-\mathrm{C}_{18}, \mathrm{C}_{18: 1}, \mathrm{C}_{18: 2}$ & $\begin{array}{l}\text { Alkaline hot springs of Lake } \\
\text { Bogoria, Kenya }\end{array}$ & $\begin{array}{l}\text { Svetlitshnyi et al. } \\
\quad \text { (1996) }\end{array}$ \\
\hline $\begin{array}{l}\text { Thermosyntropha } \\
\text { tengcongensis }\end{array}$ & $\begin{array}{l}\text { Methanothermobacter } \\
\text { thermautotrophicus }\end{array}$ & $\mathrm{C}_{4}-\mathrm{C}_{18}, \mathrm{C}_{18: 1}, \mathrm{C}_{18: 2}$ & $\begin{array}{l}\text { Hot spring in Yunnan province, } \\
\text { China }\end{array}$ & Zhang et al. (2012) \\
\hline
\end{tabular}

aThe number of carbon atoms of the fatty acids and the position of the double bond are indicated.

Syntrophus aciditrophicus, a Gram-negative benzoatedegrading bacterium that it is also able to degrade medium- and long-chain fatty acids in co-culture with a methanogen (Jackson et al. 1999). Thermophilic syntrophic butyrate-degrading bacteria are Thermosyntropha lipolytica (Svetlitshnyi et al. 1996) and Syntrophothermus lipocalidus (Sekiguchi et al. 2000). None of the fatty acid-degrading bacteria that grow syntrophically with methanogens has been described to reduce sulfate. Most fatty acid degraders are able to ferment crotonate, which was used to obtain pure cultures. However, Syntrophomonas sapovorans and S. zehnderi are not able to ferment crotonate, and are only available in syntrophic methanogenic co-cultures (Roy et al. I986; Sousa et al. 2007).

Butyrate and longer chain fatty acids are degraded via so-called $\beta$-oxidation (Schink \& Stams 2006; McInerney et al. 2008). In a series of reactions acetyl groups are cleaved off yielding acetate, hydrogen and formate. To metabolize fatty acids, first activation to a HS-CoA derivative takes place. The HS-CoA-derivative is then dehydrogenated to form an enoyl-CoA. After water addition, a second dehydrogenation takes place to form a ketoacylacetyl-CoA. After hydrolysis acetyl-CoA and an acyl-CoA are formed, which enters another cycle of dehydrogenation and the cleaving off of acetyl-CoA.

\section{EFFECT OF GRANULATION}

The formation of granular sludge is one of the reasons for the success of anaerobic treatment of wastewaters (Kosaric \& Blaszczyk 1990; Lettinga 1995). In particular, aggregation of syntrophic communities is essential to achieve high rates of methanogenesis with propionate and butyrate. Figure 1 illustrates the dense packing of microorganisms and microcolonies in the granule structure. As pointed out by 
Table 3 Detection and quantification of syntrophic bacteria in anaerobic reactors

\begin{tabular}{|c|c|c|c|c|}
\hline Reactor type & Reactor feed and operational conditions & Syntrophic bacteria abundance & Detection/Quantification method & Reference \\
\hline $\begin{array}{l}\text { Mesophilic laboratory-scale } \\
\text { UASB reactor }\end{array}$ & $\begin{array}{l}\text { Industrial wastewater from a brewery } \\
\mathrm{OLR}=4 \mathrm{~g}_{\mathrm{COD}} \mathrm{L}^{-1} \mathrm{day}^{-1} ; T=30^{\circ} \mathrm{C}\end{array}$ & $\begin{array}{l}\text { Syntrophobacter spp. } \\
\quad 5-10 \%\end{array}$ & FISH (probe SYN835) & $\begin{array}{l}\text { Férnandez et al. } \\
\quad(2008)\end{array}$ \\
\hline $\begin{array}{l}\text { Thermophilic full-scale } \\
\text { anaerobic reactor }\end{array}$ & $\begin{array}{l}\text { Municipal sewage sludge } \\
\qquad \mathrm{COD}_{\mathrm{in}}=59 \mathrm{~g} \mathrm{~L}^{-1} ; T=53^{\circ} \mathrm{C}\end{array}$ & $\begin{array}{l}\text { Pelotomaculum spp. } \\
\quad 0.1 \%\end{array}$ & $\begin{array}{l}\text { Real-time PCR (primers } \\
\text { DEM116f-Ih820) }\end{array}$ & $\begin{array}{l}\text { Imachi et al. } \\
\quad(2006)\end{array}$ \\
\hline $\begin{array}{l}\text { Thermophilic UASB laboratory- } \\
\text { scale reactor }\end{array}$ & $\begin{array}{l}\text { Synthetic wastewater containing sucrose, acetate, } \\
\text { propionate, and peptone or yeast extract } \\
\mathrm{COD}_{\mathrm{in}}=4 \mathrm{~g} \mathrm{~L}^{-1} ; T=55^{\circ} \mathrm{C}\end{array}$ & $\begin{array}{l}\text { Pelotomaculum spp. } \\
\quad 1.1 \% \\
\quad 0.5 \pm 0.2 \%\end{array}$ & $\begin{array}{l}\text { Real-time PCR (primers } \\
\text { DEM116f-Ih820) } \\
\text { FISH (probe Ih820) }\end{array}$ & $\begin{array}{l}\text { Imachi et al. } \\
\quad(2006)\end{array}$ \\
\hline $\begin{array}{l}\text { Thermophilic laboratory-scale } \\
\text { anaerobic reactor }\end{array}$ & $\begin{array}{l}\text { Wastewater from clear liquor manufacture } \\
\mathrm{COD}_{\mathrm{in}}=12 \mathrm{~g} \mathrm{~L}^{-1} ; T=55^{\circ} \mathrm{C}\end{array}$ & $\begin{array}{l}\text { Pelotomaculum spp. } \\
\quad 3.9 \% \\
\quad 4.1 \pm 1.1 \%\end{array}$ & $\begin{array}{l}\text { Real-time PCR (primers } \\
\text { DEM116f-Ih820) } \\
\text { FISH (probe Ih820) }\end{array}$ & $\begin{array}{l}\text { Imachi et al. } \\
\qquad(2006)\end{array}$ \\
\hline $\begin{array}{l}\text { Mesophilic UASB laboratory- } \\
\text { scale reactor }\end{array}$ & $\begin{array}{l}\text { Synthetic wastewater } \\
\qquad \operatorname{COD}_{\mathrm{in}}=2 \mathrm{~g} \mathrm{~L}^{-1} ; T=35^{\circ} \mathrm{C}\end{array}$ & $\begin{array}{l}\text { Pelotomaculum spp. } \\
\quad 0.1 \% \\
\quad<0.1 \%\end{array}$ & $\begin{array}{l}\text { Real-time PCR (primers } \\
\text { DEM116f-Ih820) } \\
\text { FISH (probe Ih820) }\end{array}$ & $\begin{array}{l}\text { Imachi et al. } \\
\quad(2006)\end{array}$ \\
\hline $\begin{array}{l}\text { Mesophilic full-scale anaerobic } \\
\text { reactor }\end{array}$ & $\begin{array}{l}\text { Municipal sewage sludge } \\
\text { COD }_{\text {in }}=58 \mathrm{~g} \mathrm{~L}^{-1} ; T=35^{\circ} \mathrm{C}\end{array}$ & $\begin{array}{l}\text { Pelotomaculum spp. } \\
\quad 0.3 \%\end{array}$ & $\begin{array}{l}\text { Real-time PCR (primers } \\
\text { DEM116f-Ih820) }\end{array}$ & $\begin{array}{l}\text { Imachi et al. } \\
\quad(2006)\end{array}$ \\
\hline $\begin{array}{l}\text { Mesophilic laboratory-scale } \\
\text { reactor (inoculated with sludge } \\
\text { from an anaerobic digester } \\
\text { from municipal wastewater } \\
\text { treatment plant) }\end{array}$ & $\begin{array}{l}\text { Synthetic wastewater made from powdered whole } \\
\text { milk } \\
\quad \mathrm{OLR}=1.5 \mathrm{~g}_{\mathrm{COD}} \mathrm{L}^{-1} \mathrm{day}^{-1} ; T=37^{\circ} \mathrm{C}\end{array}$ & $\begin{array}{l}\text { Syntrophobacter, Smithella spp. } \\
2.7-7.3 \% \\
\text { Syntrophomonadaceae detected } \\
\text { but not quantified }\end{array}$ & $\begin{array}{l}\text { MAR-FISH (probes } \\
\text { SmiSR354, SmiLR150, } \\
\text { Synbac824) } \\
\text { MAR-FISH (probe } \\
\text { Synm700) }\end{array}$ & $\begin{array}{l}\text { Ariesyady et al. } \\
\quad(2007 \mathrm{~b})\end{array}$ \\
\hline $\begin{array}{l}\text { Mesophilic full-scale anaerobic } \\
\text { digester (two reactors) }\end{array}$ & & $\begin{array}{l}\text { Syntrophobacter fumaroxidans } \\
0.27 \pm 0.14 \% / 0.37 \pm 0.12 \% \\
\text { Syntrophobacter pfennigii } \\
0.33 \pm 0.08 \% / 0.48 \pm 0.08 \% \\
\text { Syntrophobacter wolinii } \\
0.44 \pm 0.12 \% / 0.43 \pm 0.11 \% \\
\text { Smithella propionica } \\
<0.2 \% / 0.28 \pm 0.29 \% \\
\text { Syntrophomonadaceae } \\
0.42 \pm 0.16 \% / 1.76 \pm 0.25 \%\end{array}$ & $\begin{array}{l}\text { Membrane hybridization } \\
\text { (probe S.fum464) } \\
\text { Membrane hybridization } \\
\text { (probe S.pfn460) } \\
\text { Membrane hybridization } \\
\text { (probe S.wol223) } \\
\text { Membrane hybridization } \\
\text { (probe S.pro450) } \\
\text { Membrane hybridization } \\
\text { (probe Synm700) }\end{array}$ & $\begin{array}{l}\text { McMahon et al. } \\
\text { (2004) }\end{array}$ \\
\hline
\end{tabular}


Table 3 | continued

\begin{tabular}{|c|c|c|c|c|}
\hline Reactor type & Reactor feed and operational conditions & Syntrophic bacteria abundance & Detection/Quantification method & Reference \\
\hline $\begin{array}{l}\text { Mesophilic laboratory-scale } \\
\text { anaerobic digester }\end{array}$ & $\begin{array}{l}\text { Synthetic mixture of organic fraction of municipal } \\
\text { solid waste, primary sludge and waste activated } \\
\text { sludge } \\
\text { OLR }=9.4 \mathrm{~kg}_{\mathrm{vs}} \mathrm{m}^{-3} \mathrm{day}^{-1} ; T=37^{\circ} \mathrm{C}\end{array}$ & $\begin{array}{l}\text { Syntrophobacter fumaroxidans } \\
0.25 \pm 0.05 \% \\
\text { Syntrophobacter pfennigii } \\
0.67 \pm 0.07 \% \\
\text { Syntrophobacter wolinii } \\
0.47 \pm 0.06 \% \\
\text { Smithella propionica } \\
0.23 \pm 0.04 \% \\
\text { Syntrophomonadaceae } \\
0.65 \pm 0.03 \%\end{array}$ & $\begin{array}{l}\text { Membrane hybridization } \\
\text { (probe S.fum464) } \\
\text { Membrane hybridization } \\
\text { (probe S.pfn460) } \\
\text { Membrane hybridization } \\
\text { (probe S.wol223) } \\
\text { Membrane hybridization } \\
\text { (probe S.pro450) } \\
\text { Membrane hybridization } \\
\text { (probe Synm700) }\end{array}$ & $\begin{array}{l}\text { McMahon et al. } \\
\text { (200I) }\end{array}$ \\
\hline $\begin{array}{l}\text { Mesophilic full-scale anaerobic } \\
\text { digester plant }\end{array}$ & $\begin{array}{l}\text { Excess sludge of domestic wastewater treatment } \\
\text { facility } \\
\text { OLR }=2.5 \mathrm{~kg} \mathrm{~m}^{-3} \text { day }^{-1} ; T=40{ }^{\circ} \mathrm{C}\end{array}$ & $\begin{array}{l}\text { Syntrophus, Smithella } \\
\text { propionica } \\
2.0 \pm 0.7 \% \\
\text { Syntrophobacter } \\
0.5 \pm 0.4 \% \\
\text { Syntrophomonas } \\
1.5 \pm 0.5 \%\end{array}$ & $\begin{array}{l}\text { FISH (probe SmiSR354) } \\
\text { FISH (probe Synbac824) } \\
\text { FISH (probe Synm700) }\end{array}$ & $\begin{array}{l}\text { Ariesyady et al. } \\
\quad(2007 \mathrm{a})\end{array}$ \\
\hline $\begin{array}{l}\text { Mesophilic full-scale anaerobic } \\
\text { reactor }\end{array}$ & $\begin{array}{l}\text { Mixture of swine and cattle manure and a variety of } \\
\text { industrial organic waste streams }\end{array}$ & $\begin{array}{l}\text { Syntrophomonadaceae } \\
0.2-1 \% \\
\text { Syntrophomonas } \\
0.2-1 \%\end{array}$ & $\begin{array}{l}\text { Membrane hybridization } \\
\text { (probe Synm700) } \\
\text { Membrane hybridization } \\
\text { (probe Syn126) }\end{array}$ & $\begin{array}{l}\text { Hansen et al. } \\
\quad \text { (I999) }\end{array}$ \\
\hline $\begin{array}{l}\text { Mesophilic full-scale anaerobic } \\
\text { reactors (four reactors) }\end{array}$ & $\begin{array}{l}\text { Edible tallow refinery wastewater } \\
\text { Poultry slaughterhouse wastewater } \\
\text { Brewery wastewater } \\
\text { Potato processing wastewater }\end{array}$ & $\begin{array}{l}\text { Syntrophomonadaceae } \\
\quad 3 \% \\
\quad<0.01 \% \\
\quad<0.01 \% \\
\quad<0.01 \%\end{array}$ & FISH (probe Butox) & $\begin{array}{l}\text { Menes \& } \\
\quad \text { Travers (2006) }\end{array}$ \\
\hline Anaerobic lagoon & Woolscouring wastewater & $\begin{array}{l}\text { Syntrophomonadaceae } \\
\quad<0.01 \%\end{array}$ & FISH (probe Butox) & $\begin{array}{l}\text { Menes \& } \\
\quad \text { Travers (2006) }\end{array}$ \\
\hline $\begin{array}{l}\text { Mesophilic full-scale anaerobic } \\
\text { digester plant }\end{array}$ & $\begin{array}{l}\text { Excess sludge of domestic wastewater treatment } \\
\text { facility } \\
\text { OLR }=2.5 \mathrm{~kg} \mathrm{~m}^{-3} \text { day }^{-1} ; T=40^{\circ} \mathrm{C}\end{array}$ & $\begin{array}{l}\text { Syntrophus, Smithella } \\
\text { propionica } \\
2.0 \pm 0.7 \% \\
\text { Syntrophobacter } \\
0.5 \pm 0.4 \% \\
\text { Syntrophomonas } \\
1.5 \pm 0.5 \%\end{array}$ & $\begin{array}{l}\text { FISH (probe SmiSR354) } \\
\text { FISH (probe Synbac824) } \\
\text { FISH (probe Synm700) }\end{array}$ & $\begin{array}{l}\text { Ariesyady et al. } \\
\quad(2007 \mathrm{a})\end{array}$ \\
\hline
\end{tabular}

OLR: Organic loading rate; COD: Chemical oxygen demand; FISH: Fluorescence in situ hybridization; MAR-FISH: Microautoradiography combined with FISH; Real-time PCR: Real-time polymerase chain reaction. 
Schink \& Thauer (I988) by bringing hydrogen-producing bacteria into close proximity with hydrogen-consuming methanogens the distance that hydrogen has to diffuse from the producing to the consuming microorganisms becomes shorter, and this results in higher conversion rates. Thus, the compact microbial structure in methanogenic granular sludge is functional. It has been observed that when the structure is disrupted the specific methanogenic activity with propionate decreases considerably, while the specific methanogenic activity with acetate is not affected (Grotenhuis et al. I99I; Schmidt \& Ahring 1995).

Another factor that affects the specific activity is the size of the granules. When granules are too large, the centre of the granules will become substrate limited. Moreover, biogas may accumulate in the granules that are weakened in structure inside. This may cause a disintegration of the mature granules into smaller particles that grow again. In this way, the average size of the granules is determined by the wastewater composition.

In the granules competition for space takes place as well. To efficiently degrade fatty acids, the microorganisms in the granule should mainly consist of acetogenic fatty aciddegrading bacteria and acetoclastic and hydrogenotrophic methanogens. Fluorescence in situ hybridization (FISH) has been used to study the structural organization of anaerobic granular sludge (Harmsen et al. I996; Sekiguchi et al. I999). In these studies, a close association between Syntrophobacter species and hydrogenotrophic methanogens belonging to the Methanomicrobiales group has been observed. Nevertheless, microbial community analysis with molecular techniques has shown that granular sludge of full-scale reactors rarely harbours high numbers of acetogenic bacteria (Table 3). This suggests that fatty acid-degrading communities establish high specific conversion rates, and that even higher conversion rates can be achieved by minimizing the numbers of primary fermenters in the granules.

Still, due to the limitations in quantification of individual groups of bacteria in mixed anaerobic biomass, fluxes can hardly be attributed to concentrations of specific microorganisms. Consequently, conversion rates are expressed per amount of lumped biomass in terms of volatile suspended solids (VSS). Herewith, it remains unclear if energy is shared equally between the partner microorganisms and what the mutual effects of different acetogenic substrates are on their degradation. Detailed quantitative insight is required about the electron fluxes between the partner organisms in anaerobic sludges and the way they share the chemical energy available in the conversion of substrates. Relatively novel molecular biology-based tools allow for quantification of different trophic groups in anaerobic environments and combined with flux measurements will allow for a detailed understanding of anaerobic conversion processes. Based on the improved understanding of electron transfer in anaerobic environments it can readily be envisioned that the anaerobic digestion process can still be improved significantly.

\section{CONCLUSIONS}

High-rate methanogenesis is required to treat industrial wastewaters efficiently and conserve the chemical energy in the organic pollutants as biogas. In the complete conversion of organic pollutants to methane and carbon dioxide, syntrophic methanogenic communities of bacteria and archaea play an indispensable role. Both Gram-negative (deltaproteobacteria) and Gram-positive bacteria with the ability to grow with propionate and butyrate in syntrophy with methanogens have been described. Some of the described bacteria have been obtained in pure culture, while others are only maintained in syntrophic co-culture with a defined methanogen. In high-rate methanogenic bioreactors propionate-degrading and butyrate-degrading acetogenic bacteria form microcolonies with methanogens. The short distances between the bacteria and methanogens are favourable for transfer of metabolites from bacteria to archaea and result in high conversion rates. To further improve the rate of methane formation with mixtures of fatty acids as substrates the underlying mechanisms of interspecies electron transfer in syntrophic communities need to be better understood. In addition, insight is needed into how by process engineering the compact structure of syntrophic communities in granules can be obtained and maintained.

\section{ACKNOWLEDGEMENT}

Our research is funded by grants from the division of Chemical Sciences (CW) and Earth and Life Sciences (ALW) of The Netherlands Organisation for Scientific Research (NWO) and by the Technology Foundation (STW), the applied science division of NWO.

\section{REFERENCES}

Ariesyady, H. D., Ito, T. \& Okabe, S. 2007a Functional bacterial and archaeal community structures of major trophic groups in a full-scale anaerobic digester. Water Res. 41, 1551-1568. 
Ariesyady, H. D., Ito, T., Yoshiguchi, K. \& Okabe, S. 2007b Phylogenetic and functional diversity of propionate-oxidizing bacteria in an anaerobic digester sludge. Appl. Environ. Biotechnol. 75, 673-683.

de Bok, F. A. M., Harmsen, H. J. M., Plugge, C. M., de Vries, M. C., Akkermans, A. D. L., de Vos, W. M. \& Stams, A. J. M. 2005 The first true obligately syntrophic propionate-oxidizing bacterium, Pelotomaculum schinkii sp. nov., co-cultured with Methanospirillum hungatei, and emended description of the genus Pelotomaculum. Int. J. Syst. Evol. Microbiol. 55, 1697-1703.

de Bok, F. A. M., Plugge, C. M. \& Stams, A. J. M. 2004 Interspecies electron transfer in methanogenic propionate degrading consortia. Water Res. 38, 1368-1375.

de Bok, F. A. M., Stams, A. J. M., Dijkema, C. \& Boone, D. R. 2001 Pathway of propionate oxidation by a syntrophic culture of Smithella propionica and Methanospirillum hungatei. Appl. Environ. Microbiol. 67, 1800-1804.

Boone, D. R. \& Bryant, M. P. I980 Propionate-degrading bacterium, Syntrophobacter wolinii sp. nov., gen. nov. from methanogenic ecosystems. Appl. Environ. Microbiol. 40, 626-632.

Chen, S. Y., Liu, X. L. \& Dong, X. Z. 2005 Syntrophobacter sulfatireducens sp. nov., a novel syntrophic, propionateoxidizing bacterium isolated from UASB reactors. Int. J. Syst. Evol. Microbiol. 55, 1319-1324.

Conrad, R. I999 Contribution of hydrogen to methane production and control of hydrogen concentration in methanogenic soils and sediments. FEMS Microbiol. Ecol. 28, 193-202.

Férnandez, N., Díaz, E. E., Amils, R. \& Sanz, J. L. 2008 Analysis of microbial community during biofilm development in an anaerobic wastewater treatment reactor. Microbial Ecol. 56, 121-132.

Franklin, R. J. 200I Full scale experience with anaerobic treatment of wastewater. Water Sci. Technol. 44 (8), 1-6.

Grotenhuis, J. T. C., Smit, M., Plugge, C. M., Xu, Y., Van Lammeren, A. A. M., Stams, A. J. M. \& Zehnder, A. J. B. I99I Bacteriological composition and structure of granular sludge adapted to different substrates. Appl. Environ. Microbiol. 57, 1942-1949.

Gujer, W. \& Zehnder, A. J. B. 1983 Conversion processes in anaerobic digestion. Water Sci. Technol. 15, 127-167.

Hansen, K. H., Ahring, B. K. \& Raskin, L. 1999 Quantification of syntrophic fatty acid- $\beta$-oxidizing bacteria in a mesophilic biogas reactor by oligonucleotide probe hybridization. Appl. Environ. Microbiol. 65, 4767-4774.

Harmsen, H. J. M., Kengen, H. M. P., Akkermans, A. D. L., Stams, A. J. M. \& de Vos, W. M. I996 Detection and localization of syntrophic propionate-oxidizing bacteria in granular sludge by in situ hybridization using 16S rRNA-based oligonucleotide probes. Appl. Environ. Microbiol. 62, 1656-1663.

Harmsen, H. J. M., Van Kuijk, B. L. M., Plugge, C. M., Akkermans, A. D. L., de Vos, W. M. \& Stams, A. J. M. 1998 Syntrophobacter fumaroxidans sp. nov., a syntrophic propionate-degrading sulfate-reducing bacterium. Int. J. Syst. Bacteriol. 48, 1383-1387.
Hatamoto, M., Imachi, H., Fukayo, S., Ohashi, A. \& Harada, H. 2007 Syntrophomonas palmitatica sp. nov., an anaerobic, syntrophic, long-chain fatty-acid-oxidizing bacterium isolated from methanogenic sludge. Int. J. Syst. Evol. Microbiol. 57, 2137-2142.

Hattori, S. 2008 Syntrophic acetate-oxidizing microbes in methanogenic environments. Microbes Environ. 23, 118-127.

Imachi, H., Sekiguchi, Y., Kamagata, Y., Hanada, S., Ohashi, A. \& Harada, H. 2002 Pelotomaculum thermopropionicum gen. nov., sp. nov., an anaerobic, thermophilic, syntrophic propionate-oxidizing bacterium. Int. J. Syst. Evol. Microbiol. 52, 1729-1735.

Imachi, H., Sekiguchi, Y., Kamagata, Y., Loy, A., Qiu, Y.-L., Hugenholtz, P., Kimura, N., Wagner, M., Ohashi, A. \& Harada, H. 2006 Non-sulfate-reducing, syntrophic bacteria affiliated with Desulfotomaculum cluster I are widely distributed in methanogenic environments. Appl. Environ. Microbiol. 72, 2080-2091.

Imachi, H., Sakai, S., Ohashi, A., Harada, H., Hanada, S., Kamagata, Y. \& Sekiguchi, Y. 2008 Pelotomaculum propionicicum sp. nov., an anaerobic, mesophilic, obligately syntrophic, propionate-oxidizing bacterium. Int. J. Syst. Evol. Microbiol. 57, 1487-1492.

Jackson, B. E., Bhupathiraju, V. K., Tanner, R. S., Woese, C. R. \& McInerney, M. J. 1999 Syntrophus aciditrophicus sp. nov., a new anaerobic bacterium that degrades fatty acids and benzoate in syntrophic association with hydrogen-using microorganisms. Arch Microbiol. 171, 107-114.

Kendall, M. M., Liu, Y. \& Boone, D. R. 2006 Butyrate- and propionate-degrading syntrophs from permanently cold marine sediments in Skan Bay, Alaska, and description of Algorimarina butyrica gen. nov., sp. nov. FEMS Microbiol. Lett. 262, 107-114.

Kosaric, N. \& Blaszczyk, R. I99o Microbial aggregates in anaerobic wastewater treatment. Adv. Biochem. Eng. Biotechnol. 42, 27-62.

Lettinga, G. 1995 Anaerobic digestion and wastewater treatment systems. Antonie van Leeuwenhoek 67, 3-28.

Lettinga, G., van Velsen, A. F. M., Hobma, S. W., De Zeeuw, W. \& Klapwijk, A. I98o Use of the Upflow Sludge Blanket (USB) reactor concept for biological wastewater treatment, especially for anaerobic treatment. Biotechnol. Bioeng. 22, 699-734.

van Lier, J. B., Van der Zee, F. P., Rebac, S. \& Kleerebezem, R. 200I Advances in high rate anaerobic treatment: staging of reactor systems. Water Sci. Technol. 44 (8), 15-20.

Liu, Y., Balkwill, D. L., Aldrich, H. C., Drake, G. R. \& Boone, D. R. I999 Characterization of the anaerobic propionatedegrading syntrophs Smithella propionica gen. nov., sp. nov. and Syntrophobacter wolinii. Int. J. Syst. Bacteriol. 49, 545-556.

Lorowitz, W. H., Zhao, H. X. \& Bryant, M. P. 1989 Syntrophomonas wolfei subsp saponavida subsp. nov., a long chain fatty-acid degrading, anaerobic, syntrophic bacterium Syntrophomonas wolfei subsp. wolfei subsp. nov. - and emended descriptions of the genus and species. Int. J. Syst. Bacteriol. 39, 122-126. 
Macarie, H. 2000 Overview of the application of anaerobic treatment to chemical and petrochemical wastewaters. Water Sci. Technol. 42 (5-6), 201-213.

Mackie, R. I. \& Bryant, M. P. I98I Metabolic activity of fatty acid-oxidizing bacteria and the contribution of acetate, propionate, butyrate, and $\mathrm{CO}_{2}$ to methanogenesis in cattle waste at 40 and $60^{\circ} \mathrm{C}$. Appl. Environ. Microbiol. 41, 1363-1373.

McInerney, M. J., Bryant, M. P., Hespell, R. B. \& Costerton, J. W. I98I Syntrophomonas wolfei gen. nov. sp. nov., an anaerobic, syntrophic, fatty-acid oxidizing bacterium. Appl. Environ. Microbiol. 41, 1029-1039.

McInerney, M. J., Bryant, M. P. \& Pfennig, N. I979 Anaerobic bacterium that degrades fatty-acids in syntrophic association with methanogens. Arch. Microbiol. 122, 129-135.

McInerney, M. J., Struchtemeyer, C. G., Sieber, J., Mouttaki, H., Stams, A. J. M., Schink, B., Rohlin, L. \& Gunsalus, R. P. 2008 Physiology, ecology, phylogeny, and genomics of microorganisms capable of syntrophic metabolism. Ann. N. Y. Acad. Sci. 1125, 58-72.

McMahon, K. D., Stroot, P. G., Mackie, R. I. \& Raskin, L. 200I Anaerobic co-digestion of municipal solid waste and biosolids under various mixing conditions - II: microbial population dynamics. Water Res. 35, 1817-1827.

McMahon, K. D., Zheng, D., Stams, A. J. M., Mackie, R. I. \& Raskin, L. 2004 Microbial population dynamics during startup and overload conditions of anaerobic digesters treating municipal solid waste and sewage sludge. Biotechnol. Bioeng. 87, 823-834.

Menes, R. J. \& Travers, D. 2006 Detection of fatty acid betaoxidizing syntrophic bacteria by fluorescence in situ hybridization. Water Sci. Technol. 54, 33-39.

Mountfort, D. O. \& Bryant, M. P. I982 Isolation and characterization of an anaerobic benzoate-degrading bacterium from sewage sludge. Arch. Microbiol. 133, 249-256.

Müller, N., Worm, P., Schink, B., Stams, A. J. M. \& Plugge, C. M. 2010 Syntrophic butyrate and propionate oxidation processes: from genomes to reaction mechanisms. Environ. Microbiol. Reports 2, 489-499.

Nilsen, R. K., Torsvik, T. \& Lien, T. 1996 Desulfotomaculum thermocisternum sp. nov., a sulfate reducer isolated from a hot North Sea oil reservoir. Int. J. Syst. Bacteriol. 46, 397-402.

Plugge, C. M., Balk, M. \& Stams, A. J. M. 2002 Desulfotomaculum thermobenzoicum subsp. thermosyntrophicum subsp. nov., a thermophilic, syntrophic, propionate-oxidizing, sporeforming bacterium. Int. J Syst. Evol. Microbiol. 52, 391-399.

Roy, F., Samain, E., Dubourguier, H. C. \& Albagnac, G. 1986 Syntrophomonas sapovorans sp. nov., a new obligately proton reducing anaerobe oxidizing saturated and unsaturated long chain fatty acids. Arch. Microbiol. 145, 142-147.

Schink, B. \& Stams, A. J. M. 2006 Syntrophism among prokaryotes. In: The Prokaryotes: an Evolving Electronic Resource for the Microbiological Community (M. Dworkin, S. Falkow, E. Rosenberg, K.-H. Schleifer \& E. Stackebrandt, eds). Springer-Verlag, New York, pp. 309-335.
Schink, B. \& Thauer, R. K. 1988 Energetics of syntrophic methane formation and the influence of aggregation. In: Granular Anaerobic Sludge; Microbiology and Technology (G. Lettinga, A. J. B. Zehnder, J. T. C. Grotenhuis \& L. W. Hulshoff Pol, eds), Pudoc, Wageningen, The Netherlands, pp. 5-17.

Schmidt, J. E. \& Ahring, B. K. 1995 Interspecies electron transfer during propionate and butyrate degradation in mesophilic, granular sludge. Appl. Environ. Microbiol. 61, 2765-2767.

Sekiguchi, Y., Kamagata, Y., Nakamura, K., Ohashi, A. \& Harada, H. I999 Fluorescence in situ hybridization using 16S rRNAtargeted oligonucleotides reveals localization of methanogens and selected uncultured bacteria in mesophilic and thermophilic sludge granules. Appl. Environ. Microbiol. 65, 1280-1288.

Sekiguchi, Y., Kamagata, Y., Nakamura, K., Ohashi, A. \& Harada, H. 2000 Syntrophothermus lipocalidus gen. nov., sp. nov., a novel thermophilic, syntrophic, fatty-acid-oxidizing anaerobe which utilizes isobutyrate. Int. J. Syst. Evol. Microbiol. 2, 771-779.

Sousa, D. Z., Smidt, H., Alves, M. M. \& Stams, A. J. M. 2007 Syntrophomonas zehnderi sp. nov., an anaerobe that degrades long chain fatty acids in co-culture with Methanobacterium formicicum. Int. J. Syst. Evol. Microbiol. 57, 609-615.

Sousa, D. Z., Smidt, H., Alves, M. M. \& Stams, A. J. M. 2009 Degradation of saturated and unsaturated long-chain fatty acids by syntrophic methanogenic communities. FEMS Microbiol. Ecol. 68, 257-272.

Stams, A. J. M. 1994 Metabolic interactions between anaerobic bacteria in methanogenic environments. Antonie van Leeuwenhoek 66, 271-294.

Stams, A. J. M. \& Plugge, C. M. 2009 Electron transfer in syntrophic communities of anaerobic bacteria and archaea. Nature Rev. Microbiol. 7, 568-577.

Stams, A. J. M., de Bok, F. A. M., Plugge, C. M., van Eekert, M. H. A., Dolfing, J. \& Schraa, G. 2006 Exocellular electron transfer in anaerobic microbial communities. Environ. Microbiol. 8, 371-382.

Stieb, M. \& Schink, B. 1985 Anaerobic oxidation of fatty-acids by Clostridium bryantii sp. nov., a sporeforming, obligately syntrophic bacterium. Arch. Microbiol. 140, 387-390.

Svetlitshnyi, V., Rainey, F. \& Wiegel, J. 1996 Thermosyntropha lipolytica gen. nov., sp. nov., a lipolytic, anaerobic, alkalitolerant, thermophilic bacterium utilizing short- and long-chain fatty acids in syntrophic coculture with a methanogenic archaeum. Int. J. Syst. Bacteriol. 46, 1131-1137.

Thauer, R. K., Jungermann, K. \& Decker, K. I977 Energy conservation in chemotrophic anaerobic bacteria. Bacteriol. Rev. 41, 100-180.

Wallrabenstein, C., Hauschild, E. \& Schink, B. 1995 Syntrophobacter pfennigii sp. nov., new syntrophically propionate-oxidizing anaerobe growing in pure culture with propionate and sulfate. Arch. Microbiol. 164, 346-352.

Westerholm, M., Dolfing, J., Sherry, A., Gray, N. D., Head, I. A. \& Schnürer, A. 20II Quantification of syntrophic acetateoxidizing microbial communities in biogas processes. Environ. Microbiol. Rep. 3, 500-505. 
Westerholm, M., Roos, S. \& Schnürer, A. 20Io Syntrophaceticus schinkii gen. nov., sp. nov., ananaerobic, syntrophic acetateoxidizing bacterium isolated from a mesophilic anaerobic filter. FEMS Microbiol. Lett. 309, 100-104.

Worm, P., Stams, A. J. M., Cheng, X. \& Plugge, C. M. 20II Growth and substrate dependent transcription of formate dehydrogenase and hydrogenase coding genes in Syntrophobacter fumaroxidans and Methanospirillum hungatei. Microbiology 157, 280-289.

Wu, C. G., Liu, X. L. \& Dong, X. Z. 2006a Syntrophomonas cellicola sp. nov., a spore forming syntrophic bacterium isolated from a distilled-spirit-fermenting cellar, and assignment of Syntrophospora bryantii to Syntrophomonas bryantii comb. nov. Int. J. Syst. Evol. Microbiol. 56, 2331-2335.

Wu, C., Liu, X. L. \& Dong, X. Z. 2006b Syntrophomonas erecta subsp. sporosyntropha subsp. nov., a spore-forming bacterium that degrades short chain fatty acids in co-culture with methanogens. Syst. Appl. Microbiol. 29, 457-462.

Wu, C. G., Dong, X. Z. \& Liu, X. L. 2007 Syntrophomonas wolfei subsp. methylbutyratica subsp. nov., and assignment of
Syntrophomonas wolfei subsp. saponavida to Syntrophomonas saponavida sp. nov. comb. nov. Syst. Appl. Microbiol. 30, 376-380.

Zhang, C. Y., Liu, X. L. \& Dong, X. Z. 2004 Syntrophomonas curvata sp. nov., an anaerobe that degrades fatty acids in co-culture with methanogens. Int. J. Syst. Evol. Microbiol. 54, 969-973.

Zhang, C. Y., Liu, X. L. \& Dong, X. Z. 2005 Syntrophomonas erecta sp. nov., a novel anaerobe that syntrophically degrades short-chain fatty acids. Int. J. Syst. Evol. Microbiol. 55, 799-803.

Zhang, F., Liu, X. \& Dong, X. Z. 2012 Thermosyntropha tengcongensis sp. nov., a thermophilic bacterium that syntrophically degrades long-chain fatty acids. Int. J. Syst. Evol. Microbiol. 62 (4), 759-763.

Zhao, H. X., Yang, D. C., Woese, C. R. \& Bryant, M. P. 1990 Assignment of Clostridium bryantii to Syntrophospora bryantii gen. nov., comb. nov. on the basis of a $16 \mathrm{~S}$ ribosomal-RNA sequence analysis of its crotonate-grown pure culture. Int. J. Syst. Bacteriol. 40, 40-44.

First received 16 September 2011; accepted in revised form 5 March 2012 